\title{
Optimal Node Scheduling for Effective Energy Usage in Sensor Networks
}

\author{
A. Srivastava, J. Sobaje, M. Potkonjak, and M. Sarrafzadeh \\ Computer Science Department, University of California, Los Angeles, CA 90095-1596 \\ \{apple, jsobaje, miodrag, majid\}@cs.ucla.edu
}

\begin{abstract}
Up until now, low power system approaches have been restricted to single physical systems. The recent emergence of distributed embedded systems has created a need for power optimization across individual system boundaries. In particular, a great deal of excitement has been generated by wireless ad-hoc sensor networks, which integrate communication, computation, and sensing elements into self-organizing, adaptive, and multi-functional systems.

We address power management in these types of systems. We focus our attention on the problem of node scheduling of a minimum degree of coverage. We have developed provably optimal polynomial time algorithms. Furthermore, we analyze the scaling properties of the problem as the number of sensors in the network increase. Extensive simulations provide a number of interesting and important insights into power consumption trade-offs in sensor networks.
\end{abstract}

\section{Introduction}

Wireless ad-hoc sensor networks are a prime example of a second generation distributed system. On one end, they provide an interface to the widely used Internet. On the other end they provide an interface to the real world. It has been firmly established that, at least now and in the near future, the limiting factor for effective use of adhoc sensor networks is power consumption [Rab00]. A power managed design methodology for systems based on sensor networks is hence imperative. It would enable the design of network systems with high longevity and stable quality of service. In this paper, we address the problem of scheduling the nodes to be turned on such that the overall power dissipation is controlled and minimized while achieving a minimum degree of region coverage (defined later). We solve this problem optimally using min-cost flow formulation.

There are many tasks that sensor networks could perform, some of which are fundamental to many applications. One such task is the problem of finding a path of nodes from one side to the other. This path of nodes that are "turned on" could have many applications. Establishing a minimum level communication between one side to the other, detecting objects trying to cross the region are a few to name. Other applications like military, traffic monitoring etc will also extensively use this path of nodes. Hence a methodology, which controls the power dissipation while picking up the nodes that form this path will greatly affect the power dissipation of the application.

In this paper we optimally solve the problem of scheduling the nodes to be switched on such that there exists at-least one path from one side to the other at every time instance and the overall power dissipation is minimized. Since the problem is solved optimally, at first glance it may seem that there is no need for experimental evaluation. However, the key question in wireless ad-hoc sensor networks is how well the algorithms scale. In order to address such concerns, we studied the behavior of algorithms as the size of the network in terms of used sensors increases.

The remainder of the paper is organized as follows. Section 2 describes the related work in sensor networks, power optimization, and relevant statistical and combinatorial techniques. Preliminary technical information along with formal problem definition is discussed in Section 3. Section 4 contains the technical core of the paper: optimal polynomial time algorithms for power minimization during object detection procedures. Finally, before conclusion, we present experimental results related to our statistical studies on algorithms scalability.

\section{Related Work}

\subsection{Ad-hoc Sensor Networks}

Due to rapid development in technology, wireless networks have become feasible and cost effective. Wireless networks can have many attractive flavors. On one end of the spectrum, there are multimedia and gigabit wireless LANs, where the dominant issue is high bandwidth. On the other end of the spectrum are the last ten meters (wireless connection between computers and peripherals), last one meter (sensor networks) and last inch (personal and implantable networks).

It is projected that the Bluetooth (a protocol for communication in wireless networks) [Blu00] devices will form a $\$ 5$ billion annual market in less than three years. A number of applications are envisioned for these types of networks that can dramatically alter the human life [Ten00] [Est00]. A number of challenging technical problems are associated with wireless ad-hoc networks in general and sensor networks in particular. They include a need for new types of signal processing [Yao98] [Pot00], operating systems features [Adj99], selforganization and deployment [Agr99], low power design [Rab00], integration [Bul96], and issues related to embedded systems [Bor00].

\subsection{Power Modeling, Minimization and Management}

Power is an important optimization metric both while designing the sensor nodes and while developing algorithms to solve specific problems on these networks. A lot of research has gone into the development of design methodologies and systems for low power applications. [Ang96] contains a survey of low power techniques for digital circuits. [Ped99] considers the problem of maximizing battery life in CMOS circuits. For wireless systems, power issues at circuit level are addressed in [Abi00] and at architectural level in [Rab00a]. On the algorithmic side, [Sin98] discusses new power aware metrics for determining routes in traditional wireless ad-hoc networks. Variation of transmitter power level of a mobile node in a wireless network for fixed quality of service in varying channel interference is discussed in [Ru197]. In this paper we discuss provably optimal algorithms for energy conservation while doing node scheduling.

\subsection{Statistical and Probabilistic Optimization Techniques}

Statistical techniques can be broadly divided into two groups: parametric and nonparametric [Thi88]. Parametric techniques assume that knowledge about the underlying statistical distribution is available (often normal distribution is assumed) and that the task is to validate the assumption regarding the distribution, calculate the corresponding parameters, and establish intervals of confidence [D'A86]. Nonparametric techniques do not make any assumptions about the statistical distribution. They aim to figure conceptually and quantitatively the simplest (and therefore best) model which fits the recorded data [Can99]. Therefore, nonparametric techniques are significantly more computationally intensive. In situations where the underlying statistical distribution is known, parametric techniques are attractive options. However, for object detection kind of applications, it 
is very difficult to make any assumptions about the underlying statistical distribution. Therefore, we decided to use non-parametric statistical techniques.

\section{Preliminaries}

\subsection{Sensor Networks: Architecture and Models}

Sensor Networks are networks of low power sensing devices (nodes), which are computationally rich. Networking these sensors empowers them with the ability to coordinate among themselves for solving large sensing and computational tasks. These networks would be large scale, very dynamic, and their deployment in inhospitable environments would enable easy acquisition and processing of data. There are many architectural challenges in designing such distributed systems. These sensors would be expected to coordinate among themselves and setup a network. They would have to divide tasks amongst themselves in an energy efficient fashion. Upon sensor node failure and sensor node addition, they would have to reorganize themselves. Another major constraint that these systems would have is power. Since the nodes will be battery powered, the system lifetime will be heavily dependent upon the way these systems use their available power. In this paper we discuss algorithms for efficient power management of sensor nodes.

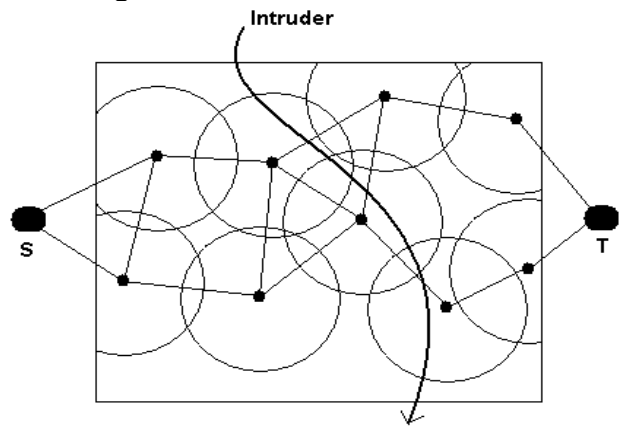

Figure 3.1: Abstraction of a Sensor Network

\subsection{Problem Formulation}

Figure 3.1 shows the abstraction of a sensor network used in this work. Each node has an associated placement coordinate and an associated radius (which could be different for different sensors), which is the radius of the circular region that the node can sense. This circle is called the region of view. We abstract this distribution of nodes to nodes in a graph $\mathrm{G}=(\mathrm{V}, \mathrm{E})$, where each graph node represents the physical sensor node. The edges in the graph can have different meanings depending on the task we are trying to achieve. For example if we want to establish a communication link between one side to the other, an edge would indicate the feasibility of having a communication between the source and destination of the edge if both are kept "turned on ". If we are monitoring traffic, an edge would indicate the capability of the system to detect any movement between the source and destination nodes.

In this paper, we try to find a path from left to right edge of the region. These nodes will be "turned on" and hence will dissipate expensive system energy. Keeping the same path activated all the time is not a very good idea from the overall system energy and lifetime point of view. The problem at hand is to schedule the nodes to be turned on such that there exists exactly one path at any instance of time and overall energy dissipation is also balanced out. More formally the problem can be described as follows

Given a graph $\mathrm{G}=(\mathrm{V}, \mathrm{E})$ with a source $\mathrm{S}$ and a destination $\mathrm{T}$. Let us assume that each time step comprises of some pre-specified number of time units (say Ds) that does not change. We want to find a path from $\mathrm{S}$ to $\mathrm{T}$ for $\mathrm{K}$ such time steps. Nodes $\mathrm{S}$ and $\mathrm{T}$ indicate the external basestations on the sides of the region between which the path needs to be established. Each node has an associated energy Ei and power dissipated per unit time step Pi. Hence the quantity Ei/Pi indicates the number of time steps which this node can be "turned on" without exhausting its energy reserves. This quantity will be called node capacity in the subsequent paragraphs. Each node also has an associated cost which signifies the power dissipated per unit time step to keep it on. The objective is to schedule the nodes to be turned on in such a way that in every time step there is exactly one path from $\mathrm{S}$ to $\mathrm{T}$ and the overall system lifetime is maximized. This needs to be for $\mathrm{K}$ time steps. We believe that the following metric is a good indicator of the overall system lifetime.

\section{Lifetime $=\Sigma \mathbf{E} i$}

In order to maximize the Lifetime, we would like to pick nodes with lesser cost. After K time-steps, the system lifetime will be given by $\Sigma(\mathrm{Ei}$ - $\mathrm{Pi} *$ ni) where $\mathrm{Pi}$ is the energy dissipated per time step and ni is the number of times a node is kept on (assuming the overhead of turning on and off is negligible).

$$
\begin{gathered}
\operatorname{Maximize} \Sigma(\mathbf{E i}-\mathbf{P i} * \mathbf{n i}) \\
=\operatorname{Minimize} \Sigma \mathbf{P i} * \mathbf{n i}
\end{gathered}
$$

Maximizing the system lifetime is equivalent to minimizing the sum of the node costs that take part in the path formation.

Problem Statement: Given a graph $G=(\mathbf{V}, \mathbf{E})$ with nodes and edges and two distinct nodes $S$ and $T$. Given node capacities $(\mathrm{Ei} / \mathrm{Pi})$ and costs $\mathrm{Pi}$, schedule the nodes in $\mathrm{K}$ time slots such that

- In each time slot there exists exactly one path of nodes that make a connected path from $S$ to $T$

- The sum of the costs of the nodes that take part in this process is minimized.

- Each node that is scheduled in a time slot must have enough energy to remain working for the entire time slot.

We solve this problem optimally using the min-cost K-flow algorithm.

\subsection{Combinatorial Optimization Techniques}

In this paper we use the following combinatorial optimization techniques: shortest path and mincost flow.

\subsubsection{Shortest Path Problem}

The shortest path problem is the problem of finding a path from a node in a graph to another node. This path should have the shortest length, or weight if edge weights are defined. This problem has been extensively studied [Cor90]. We use the breath first search algorithm [Moo59] to find the shortest path. This algorithm proceeds by expanding the frontier between discovered and undiscovered vertices uniformly across the breath of the frontier. It takes $D+1$ steps to discover all vertices at distance D.

\subsubsection{The Flow Problem}

Given a network with edge capacities, maximum flow problems try to answer the following question: What is the greatest rate at which material can be shipped from source to sink without violating the capacity constraints? There are efficient algorithms to solve this problem, which are discussed extensively in [Cor90]. Basically these 
algorithms try to find a residual path from source to destination along which more flow can be pushed.

In this paper we use a modification of the conventional max-flow algorithm. The reason is that among all solutions which have the flow "f", we want to pick the solution that has the minimum cost. As we show later, we model flow as time units, and cost as energy dissipation. We intend to maximize the flow, i.e. time, but at the same time we want a minimum cost solution. Such problems come under the category of min-cost flow problems. These are discussed extensively in [For62],[Edm72].

\section{Combinatorial Optimization for Energy Minimization}

In this paper, we are trying to schedule nodes in $\mathrm{K}$ time slots such that in each time slot there exists at-least one connected path from $\mathbf{S}$ to $\mathbf{T}$. It can be seen that this amounts to finding $\mathrm{K}$ units of flow from $\mathrm{S}$ to $\mathrm{T}$ such that the node capacity constraints are met and the overall flow-cost is also minimum. It can be re-called that node capacity corresponds to the number of time slots it can be kept on without draining it's energy reserve. Node cost corresponds to amount of system energy spent for keeping it on for one time slot. Each unit of flow from $\mathrm{S}$ to $\mathrm{T}$ will correspond to a path of connected nodes.

\subsection{Maximum Temporal Coverage with Minimum Power Consumption}

In the previous sections we explained the construction of a network with from a spatial distribution of sensor nodes. Each node (except $S$ and $\mathrm{T}$ ) has an associated $\mathrm{E}_{\mathrm{i}}$ and $\mathrm{P}_{\mathrm{i}}$. We will try to solve this problem using the theory of mincost flows [For62], [Edm72]. The first requirement of any network problem is to have a network with edge capacities. The Mincost flow problem also needs non-negative cost per unit flow for each edge. The network also needs to be directed. Our abstraction of the sensor nodes generates an undirected graph having a set of nodes with positive capacities $\mathrm{C}_{\mathrm{i}}$ and costs $\mathrm{Pi}$. The first step is to make the network directed. According to [For62], this can be achieved by simply replacing each undirected edge in the parent graph by two edges pointing in opposite directions. For the sake of simplicity, let us assume that the edges coming from $\mathrm{S}$ and going into $\mathrm{T}$ were directed from the very beginning. All the edges connected to $\mathrm{S}$ point away from it and all edges connected to $\mathrm{T}$ point into it. Note that our algorithms and theory will still be valid even if these links were assumed to be undirected.

After this modification we get a directed network with node capacities and node costs. Node cost and capacity needs to be converted to edge cost and capacity. To achieve this, we transform the network as follows [For62]:

- Replicate each node $\mathrm{n}$ with nodes $\mathrm{n}$ and n'.

- Have a directed edge from $n$ to n' with capacity equal to the original node capacity.

- The cost of the new edge is same as the cost of the original node $\mathrm{n}$.

- All edges that point into the original node $\mathrm{n}$, will point into the new node $\mathrm{n}$.

- All edges that point out of the original node $n$ will point out of n'.

- All other edge capacities are infinity and edge costs are zero.

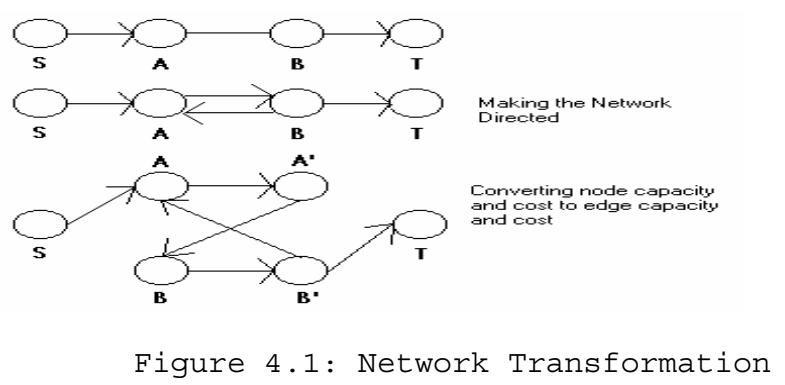

These transformations are illustrated in Figure 4.1. At this stage, we have a directed network with edge capacities and costs. We also have a number $\mathrm{K}$ that corresponds to the time units for which the path is needed. We solve this problem by augmenting $\mathrm{K}$ units of flow. Each unit of flow corresponds to a unit of time. Among all possible flows with value $\mathrm{K}$, we want the flow which has the minimum total cost. Total flow cost is defined as:

Where

$$
\text { Flow Cost }=\Sigma \mathrm{f}(\mathrm{u}, \mathrm{v}) * \mathrm{P}(\mathrm{u}, \mathrm{v})
$$

$\mathrm{f}(\mathrm{u}, \mathrm{v})$ : flow through the edge

$\mathrm{P}(\mathrm{u}, \mathrm{v})$ : Cost of the edge

Note that this number corresponds to the total power dissipated by having a path for $\mathrm{K}$ units of time. As observed in [Edm72], this problem can be solved by the theory of min-cost flows. We briefly outline the algorithm below.

\subsubsection{Min Cost Flow Algorithm}

While solving flow problems, a residual network is formed along which flow is augmented. Let us call this residual network $\mathrm{N}^{\mathrm{f}}$, where $\mathrm{f}$ is the amount of flow that is currently in the network. Associate with each edge $(\mathrm{u}, \mathrm{v})$ in the residual network a weight or cost $\delta(\mathrm{u}, \mathrm{v})$.

$$
\begin{gathered}
\delta(u, v)=P(u, v) \text { if }(u, v) € E \\
\delta(u, v)=-P(u, v) \text { if }(v, u) € E
\end{gathered}
$$

It is well known that flow algorithms proceed by augmenting flows in residual networks. Let us call this augmenting sequence by $\mathrm{f}^{0} \mathrm{f}^{1} \mathrm{f}^{2} \ldots$. In [Edm72] it is suggested that in order to solve this problem, each node $i$ must have a value $\pi(\mathrm{i})$ associated with it. The overall algorithm is enumerated below.

\section{Algorithm 1. Maximum Coverage with Minimum Power}

1. $S e t \mathrm{f}^{0}=0, \pi^{0}=0, \mathrm{i}=0$

2. Given $\mathrm{f}^{\mathrm{i}}$ and $\pi^{\mathrm{i}}$, determine $\mathrm{f}^{\mathrm{i}+1}$ by augmenting along a minimum weight path from $\mathrm{S}$ to $\mathrm{T}$ in $\mathrm{N}^{\mathrm{fi}}$ with respect to non negative weights

3. $\delta^{\mathrm{i}}(\mathrm{u}, \mathrm{v})=\pi^{\mathrm{i}}(\mathrm{u})+\delta(\mathrm{u}, \mathrm{v})-\pi^{\mathrm{i}}(\mathrm{v})$

4. If $\sigma^{\mathrm{i}}(\mathrm{u})$ indicate the weight of the shortest path from $\mathrm{S}$ to $\mathrm{u}$ w.r.t weights $\delta^{i}$ then set

5. $\pi^{\mathrm{i}+1}(\mathrm{u})=\pi^{\mathrm{i}}(\mathrm{u})+\sigma^{\mathrm{i}}(\mathrm{u})$

6. Halt when there is no augmenting path.

Theorem 1: This algorithm gives the minimum value of total flow cost for the max flow solution.

Proof : Please refer to [Edm72]

Since our original objective was to solve the problem for K units of flow, in the above algorithm we can simply stop whenever K units of 
flow are achieved. The algorithm will still give the minimum value of total flow cost for K flows [Edm72].

Now that we have the flows, the next problem is to assign paths(nodes) to time slots. This could be done arbitrarily, as the overall cost does not get affected. We propose the following algorithm to solve the problem:

\begin{tabular}{|l|}
\hline Algorithm 2: Path Construction \\
1. $\begin{array}{l}\text { Remove all edges from the network } \\
\text { that have non- positive flow. }\end{array}$ \\
2. $\begin{array}{l}\text { Find a path from } \mathrm{S} \text { to } \mathrm{T} \text { in this } \\
\text { network. Any path will work. Assign } \\
\text { this path to a time slot. }\end{array}$ \\
3. $\begin{array}{l}\text { Reduce the flow along the edges of } \\
\text { this path by one. } \\
\text { Repeat steps } 1,2 \text { and } 3 \text { until we have } \mathrm{K} \\
\text { paths. }\end{array}$ \\
\hline
\end{tabular}

Theorem 2: The algorithm outlined above always produces $\mathrm{K}$ paths from $\mathrm{S}$ to $\mathrm{T}$, if there are $\mathrm{K}$ units of flow originally in the network.

Proof: It can be seen that in every step we reduce the incoming flow of a node (which lies on the path) by one and also reduce the outgoing flow by one. So in every step we maintain the flow property, which states that the sum of incoming flow is same as outgoing flow. Hence this algorithm produces K paths.

\subsection{Interesting Simplifications}

Last section dealt with the general problem of scheduling nodes in $\mathrm{K}$ time slots. If $\mathrm{K}=1$, then the same problem can be solved by much simpler and faster algorithms. In this case the objective is to find one path from $\mathrm{S}$ to $\mathrm{T}$ with minimum cost. If all sensor nodes start with the same initial energy and require the same operating power, then the sensing path that optimizes energy consumption is simply the shortest path. The shortest path minimizes the number of nodes that must be switched on, thus minimizing the consumed energy. The existence of a path and the average length of the shortest path depend on the distribution of the sensor nodes, the number of nodes in the network, and the sensing radius of the nodes. Even if the nodes have different power dissipation levels (or different available energy), the min-cost solution can still be obtained using weighted shortest path algorithms [Cor90]. Hence the problem can be optimally solved asymptotically faster than the general case.

\subsubsection{A Different Cost Function: Maximizing the Path Lifetime}

In the previous section we used the sum of the cost of all nodes on the path as the cost of a path. A more accurate way of modeling the lifetime of a path could be to consider the minimum available energy of the nodes and picking a path which has the maximum. Hence the cost of a path $\mathrm{p}$ is

$$
\mathrm{C}_{\mathrm{p}}=\min \left(\forall(\text { nodes } \mathrm{n} \text { on path })\left(\mathrm{E}_{\mathrm{n}} / \mathrm{P}_{\mathrm{n}}\right)\right)
$$

The quantity $E_{n} / P_{n}$ denotes the number of time units that this node can be switched on (also referred to as capacity). The objective is to find a path with maximum value of $\mathrm{C}_{\mathrm{p}}$.

In order to find the path that has the maximum $\mathrm{C}_{\mathrm{p}}$, the sensor nodes $n$ in the network are sorted from highest to lowest $E_{n} / P_{n}$. A binary search is performed to obtain the solution. A subgraph is formed using only those nodes with capacity greater than the median, and the subgraph is checked to see if a sensing path exists. If a sensing path exists, the number of nodes in the subgraph is again cut in half and the process is repeated. If no sensing path exists, the number of nodes in the subgraph is expanded to include nodes with lower capacity. The minimum capacity is reported when the search is complete. The solution gives a path from $\mathrm{S}$ to $\mathrm{T}$ which has the maximum value of minimum capacity node.

\section{Experimental Results}

The previous sections described optimal algorithms to solve the single path $(\mathrm{K}=1)$ and multi-path problems. These gave a minimum cost solution where the cost metric corresponded to the total power dissipated. In order to evaluate the working of these algorithms, we made a test bed which instantiated a spatial distribution of nodes to a network problem. We assumed a $1 \mathrm{x} 1$ rectangle region in which we distributed the sensor nodes using uniform random and normal distributions. From this distribution a connected graph was initialized with nodes corresponding to the sensor nodes and edges between nodes whose region of view (section 3.2) overlap.

\subsection{Path with Minimum Power Consumption}

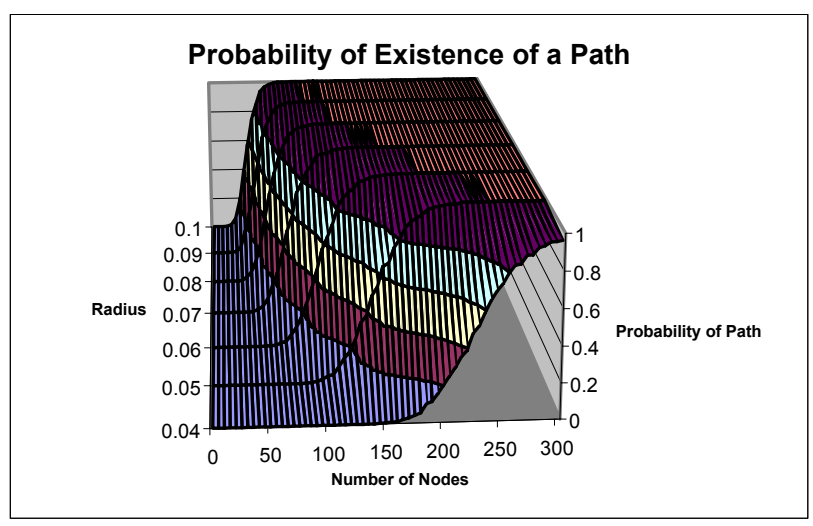

Figure 5.1 Probability of Path Existence

We first report the experimental observations for the simplified case in which $\mathrm{K}=1$. For experimental purposes the probability of existence of a path is obtained. A breadth first search is used to determine if a path from $\mathrm{S}$ to $\mathrm{T}$ exists. For a given number of sensor nodes and a given sensing radius, a large number of trials are performed in order to obtain an accurate probability. If a sensing path exists, then the breadth first search from node $S$ to node $T$ returns the length of the shortest path from $\mathrm{S}$ to $\mathrm{T}$. The average length of the shortest path is an important metric, since the amount of energy consumed by the network depends on the number of nodes that must be switched on.

Figure 5.1 shows the probability distribution of having a path from the left side of the region to the right side if the nodes are randomly distributed. The result is plotted for a uniform random distribution. The variation was shown w.r.t number of nodes and radius. The sensing radius was kept the same for all the nodes. For each value of number of nodes and radius, we got 10,000 different node distributions and computed the probability of having a path. The curve shows that if we increase the sensing radius we need lesser number of nodes to achieve the same probability. Another observation we made was that as we cut the radius in half we need approximately four times the number of 
nodes to achieve the same probability (although this may not be directly evident from this curve).

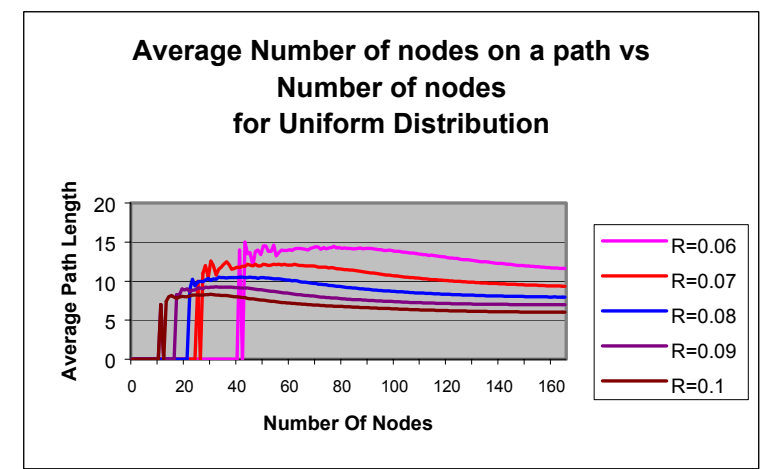

Figure 5.2 Average Number of Nodes per Path

Figure 5.2 shows the average number of nodes on the shortest path from $\mathrm{S}$ to $\mathrm{T}$ for various number of nodes and for various radii. In this experiment we set the power dissipation and radius of all nodes to the same value. Hence, the shortest path is also the shortest weighted path. We see that for the same radius, the average path length saturates to a value, beyond which addition of more nodes in the network does not help the path length. Increasing the radius does reduce this saturation point.

In section 4.2.1 we described an algorithm that finds a path which maximizes the minimum node capacity $(\mathrm{Cp}=\mathrm{En} / \mathrm{Pn}$, note that this is also the path lifetime) on the path. Figure 5.3 shows the outcome of that algorithm. For this experiment we randomly distributed the available energy. The power and radius were kept the same for each node. We see that as we increase the number of nodes and radius, the path lifetime also increases. (The values are normalized.)

\subsection{Scheduling nodes for Minimum Power Consumption}

In this section we demonstrate the results obtained by using the flow formulations described in section 4.1. The objective is to maximize the number of time units for which we can have a path (i.e. maximize $\mathrm{K})$. We study the variation of this value with different parameters.

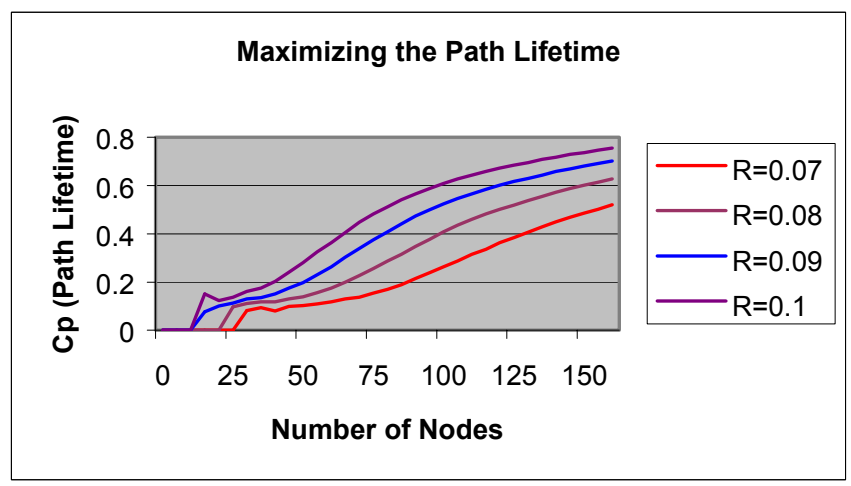

Figure 5.3 Maximizing Path Lifetime
Maxflow vs Number of Nodes and Radius Uniform Random Distribution

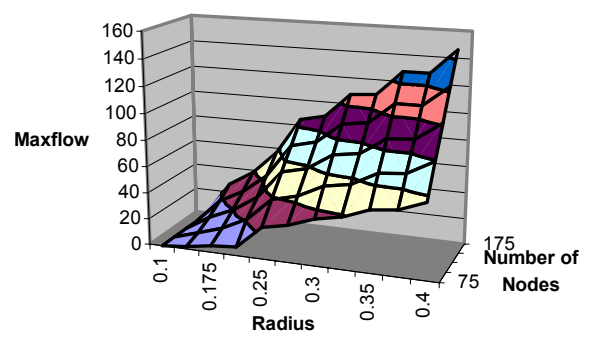

Figure 5.4 Maximizing Temporal Coverage

Figure 5.4 shows the distribution of the maximum flow with radius and nodes. Maximum Flow corresponds to the maximum number of time units that we can have a path until no path is possible. The node distribution is uniform random. The radius and power dissipation for all nodes were kept the same. The capacity (number of time units for which a node can be switched on, En/Pn) was varied randomly between 0 and a number $\mathrm{M}$ which was provided by the user. For this graph $\mathrm{M}$ was set to 10 . It can be seen that the variation is somewhat linear with radius. If we increase the number of nodes the slope of this curve increases.

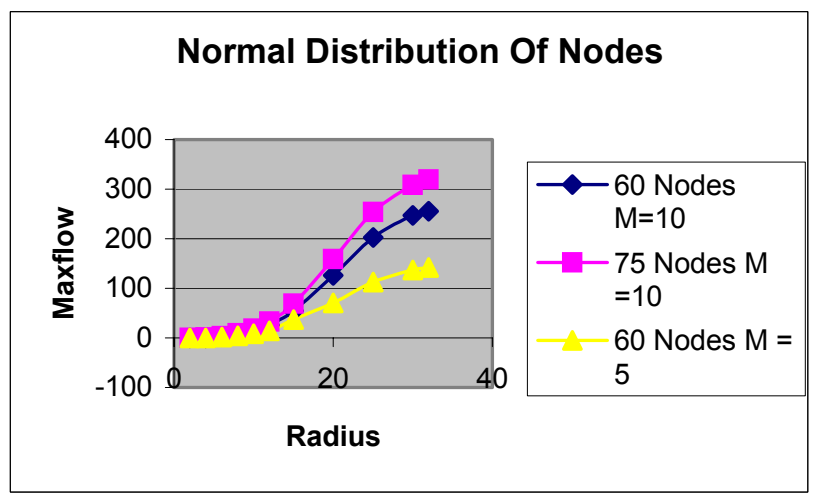

Figure 5.5: Normal Distribution

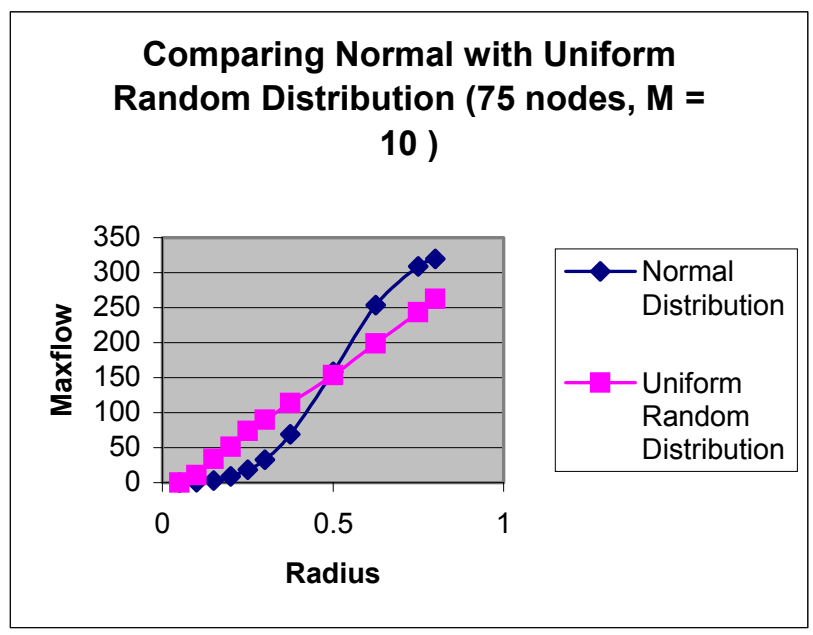

Figure 5.6: A Comparative Study 
Figure 5.5 shows the distribution of maxflow with radius for a normal distribution of sensor nodes. We see that the variation is somewhat exponential initially and then it saturates. The value at which saturation occurs increases as the nodes count increase. Again, the radius and power were kept the same for all nodes. The capacity was varied between 0 and $\mathrm{M}$ randomly. We also compared the variation of maxflow for different M. For the same number of nodes, the saturation point occurred at a smaller value of maxflow for a smaller value of M. Finally, in Figure 5.6, we compare uniform random distribution and normal distribution. Since the variation for random distribution is somewhat linear and for normal is exponential, there is a point where normal distribution begins to perform better than random. This point is clearly shown in the graph.

\section{Conclusion}

In this paper, we have addressed architectural issues regarding sensor networks. We have provided optimal algorithms to solve the node scheduling problem on a framework of sensor nodes forming a sensor network. The sensor nodes will be implemented using low power and low cost embedded systems. Power will be a primary concern in these systems as the overall system stability and lifetime depend on the power.

Through our experimental results, we have shown the behavior of the problem as the nodes scale. We found that for a particular radius there is a value for the number of nodes beyond which the pathprobability is constant. So, adding any further nodes will be wasteful. Experimental results also suggest that for a particular radius, the shortest path saturates to a value beyond which increasing the number of nodes does not help. We observed that for a uniform random distribution the maxflow keeps increasing almost linearly (with radius for a particular node count). Moreover, there is a distinct point beyond which the normal distribution starts performing better than the uniform random distribution. This happens when the value of radius is around half the linear dimension of the region.

We can conclude that sensor networks form an interesting topic for further low power research. A lot more needs to be done in terms of power optimization for such systems to be commercially viable.

\section{References}

[Abi00] A. A. Abidi, G. J. Pottie, W.J. Kaiser, "Power-conscious design of wireless circuits and systems," Proceedings of the IEEE, vol.88, pp. 1528-45, Oct. 2000.

[Adj99] W. Adjie-Winoto, E. Schwartz, H. Balakrishnan, J. Lilley. "The design and implementation of an intentional naming system". Operating Systems Review, vol.33, (no.5), Dec. 1999, pp.186-201.

[Agr99] J.R. Agre, L.P. Clare, G.J. Pottie, N.P. Romanov. "Development platform for self-organizing wireless sensor networks". Proceedings of the SPIE - The International Society for Optical Engineering, vol.3713, Apr. 1999, pp.257-68.

[And89] A.V. Goldberg, E. Tardos and R. E. Tarjan, "Network Flow Algorithms ", Technical ReportSTAN-CS-89-1252, Computer Science Department, Stanford University, 1989

[Ang96] E. de Angel, E.E. Swartzlander, "Survey of Low Power Techniques for VLSI Design”. Eighth International Conference on Innovative Systems in Silicon, Oct. 1996, pp.159-69.

[Bor00] G. Borriello, R. Want. "Embedded computation meets the World Wide Web". Communications of the ACM, Volume 43, No. 5, May. 2000, pp.59-66.

[Blu00] Bluetooth special interest group http://www.bluetooth.com/technology/
[Bul00] N. Bulusu, J. Heidemann, D. Estrin. "GPS-less Low Cost Outdoor Localization for Very Small Devices." IEEE Personal Communications, vol.7, (no.5), IEEE, Oct. 2000. pp.28-34.

[Can99] W. J. Canover. "Practical nonparametric statistics." 3rd edition. New York: Wiley, 1999.

[Cor90] T.H. Cormen, C.E. Leiserson, R.L. Rivest, "Introduction to Algorithms", MIT Press, 1990

[D'A86] R.B. D'Agostino, M. A. Stephens. "Goodness-of-fit techniques." New York: M. Dekker, 1986.

[Efr93] B. Efron, R. J. Tibshirani. "An introduction to the bootstrap." New York: Chapman \& Hall, 1993.

[Edm72] J. Edmonds, R.M. Karp, "Theoretical Improvements in Algorithmic Efficiency for Network Flow Problems". In Procs Journal of the Association for Computation Machine, Vol 19, No 2, April 1972, pp. 248-264

[Est00] D. Estrin, R. Govindan, J. Heidemann. "Embedding the Internet: introduction.". Communications of the ACM, Volume 43 , No. 5, May. 2000, pp.38-42.

[For62] L.R. Ford, D.R. Fulkerson, "Flow in Networks", Princeton University Press, 1962.

[Moo59] E. F. Moore, "The Shortest Path Through a Maze", International Symposium on Theory of Switching, Harvard University Press, 1959, pp. 285-292

[Ped99] M. Pedram, Q. Wu, "Design Considerations for BatteryPowered Electronics". In Procs Design Automation Conference1999

[Pot00] G. J. Pottie, W. J. Kaiser "Wireless integrated network sensors". Communications of the ACM, Volume 43, No. 5, May. 2000, pp. 51-58.

[Rab00] Rabaey, J.M.; et al. PicoRadio supports ad hoc ultra-low power wireless networking. Computer, July 2000, vol.33, (no.7):42-8

[Rab00a] J.M. Rabaey, "Low-power silicon architectures for wireless communications," ASP-DAC 2000, pp. 77-80, 2000.

[Rab96] Low power design methodologies / edited by Jan M. Rabaey and Massoud Pedram. Boston : Kluwer Academic Publishers, 1996

[Rul97] J. M. Rulnick, N. Bambos, "Mobile Power Management for Wireless Communication Networks", Wireless Networks, vol.3, No.1, 1997, pp.3-14.

[Sin98] S.S. Singh, M. Woo, C.S. Raghavendra, "Power-Aware Routing in Mobile Ad Hoc Networks". In Procs MOBICOM-1998

[Ten00] D. Tennenhouse. "Proactive computing." Communications of the ACM, Volume 43, No. 5, May. 2000, pp. 43-50.

[Yao98] K. Yao, R. E. Hudson, C. W. Reed, D. Chen; F. Lorenzelli. "Blind beamforming on a randomly distributed sensor array system." IEEE Journal on Selected Areas in Communications, vol.16, (no.8), IEEE, Oct. 1998, pp.1555-67. 